\title{
Situações Vivenciadas pela Equipe de Enfermagem de uma Unidade Oncológica
}

doi: https://doi.org/10.32635/2176-9745.RBC.2019v65n1.325

\author{
Situations Experienced by the Nursing Team of a Cancer Unit \\ Situaciones Vivenciadas por el Equipo de Enfermería de una Unidad Oncológica
}

\author{
Géssica Fernanda Rodrigues Monção'; Kesley de Oliveira Reticena²; Maria Fernanda Pereira Gomes³; Estela Maris Ribeiro \\ Cardoso $^{4}$; Lislaine Aparecida Fracolli ${ }^{5}$
}

Resumo

Introdução: $\mathrm{O}$ câncer é uma doença complexa que demanda cuidados e tratamentos diversos. Tendo em vista a importância da equipe de enfermagem para pacientes que sofrem com o câncer e a complexidade da atençâo e atuação nessa área, os processos de trabalho desses profissionais devem receber especial atenção. Objetivo: Compreender as situações vivenciadas por profissionais de enfermagem que cuidam de pacientes com câncer. Método: Pesquisa qualitativa realizada com 12 profissionais da equipe de enfermagem atuantes em uma unidade de oncologia de um hospital do interior do Estado de São Paulo, Brasil, durante os meses de setembro e outubro de 2017, utilizando-se um roteiro de entrevista semiestruturada contendo dados de caracterização e questôes norteadoras. Foi realizada análise temática de conteúdo, da qual emergiram as categorias "Situaçóes vivenciadas durante a prestação de cuidados na unidade oncológica" e "Sentimentos dos profissionais de enfermagem aos prestarem cuidados ao paciente com câncer". Resultados: Mesmo com situaçôes de dificuldades e sofrimentos, os profissionais demonstraram sentimentos de satisfação e gratidão por prestarem cuidados aos pacientes com câncer. Conclusáo: A motivação por parte das profissionais de enfermagem para a prestação de cuidados dignos aos pacientes com câncer supera as barreiras existentes.

Palavras-chave: Enfermagem Oncológica; Profissionais de Enfermagem; Cuidados de Enfermagem; Neoplasias.

\begin{abstract}
Introduction: Cancer is a complex disease that requires diverse care and treatment. Considering the importance of the nursing team for patients suffering from cancer and the complexity of care and performance in this area, the work processes of these professionals should receive special attention. Objective: To understand the situations experienced by nursing professionals who care for patients with cancer. Method: Qualitative research carried out with 12 nursing team professionals working in an oncology unit of a hospital in the interior of the State of São Paulo, Brazil, during the months of September and October 2017, using a semi-structured interview script characterization data and guiding questions. A thematic content analysis was carried out, from which emerged the categories "Situations experienced during care delivery in oncology unit" and "Feelings of nursing professionals to care for patients with cancer”. Results: Even with situations of difficulties and suffering, professionals showed feelings of satisfaction and gratitude for providing care to patients with cancer. Conclusion: The motivation of nursing professionals to provide decent care to cancer patients overcomes existing barriers.

Key words: Oncology Nursing; Nurse Practitioners; Nursing Care; Neoplasms.
\end{abstract}

\section{Resumen}

Introducción: El cáncer es una enfermedad compleja, que demanda cuidados y tratamientos diversos. En vista de la importancia del equipo de enfermería para pacientes que sufren con el cáncer y la complejidad de la atención y actuación en esa área, los procesos de trabajo de estos profesionales deben recibir especial atención. Objetivo: Comprender las situaciones vivenciadas por profesionales de enfermería que cuidan de pacientes con cáncer. Método: Investigación cualitativa realizada con 12 profesionales del equipo de enfermería actuantes en una unidad de oncología de un hospital del interior del Estado de São Paulo, Brasil, durante los meses de septiembre y octubre de 2017, utilizando un itinerario de entrevista semiestructurada conteniendo datos de caracterización y cuestiones orientadoras. Se realizó un análisis temático de contenido, de la cual surgieron las categorías "Situaciones vivenciadas durante la prestación de cuidados en la unidad oncológica" y "Sentimientos de los profesionales de enfermería a prestar atención al paciente con cáncer”. Resultados: Incluso con situaciones de dificultades y sufrimientos, los profesionales mostraron sentimientos de satisfacción y gratitud por prestar atención a los pacientes con cáncer. Conclusión: La motivación por parte de las profesionales de enfermería para la prestación de cuidados dignos a los pacientes con cáncer supera las barreras existentes. Palabras clave: Enfermería Oncológica; Enfermeras Practicantes; Atención de Enfermería; Neoplasias.

\footnotetext{
${ }^{1}$ Universidade Paulista (Unip). Assis (SP), Brasil. Orcid iD: https://orcid.org/0000-0002-8427-1769.

${ }^{2}$ Unip. Assis (SP), Brasil. Orcid iD: https://orcid.org/0000-0002-0905-588X

${ }^{3}$ Unip. Assis (SP), Brasil. Orcid iD: https://orcid.org/0000-0003-0577-2264

${ }^{4}$ Unip. Assis (SP), Brasil. Orcid iD: https://orcid.org/0000-0003-0989-4330

${ }^{5}$ Universidade de São Paulo (USP). São Paulo (SP), Brasil. Orcid iD: https://orcid.org/0000-0002-0936-4877

Endereço para correspondência: Kesley de Oliveira Reticena. Rua Myrtes Spera Conceição, 301 - Conjunto Nelson Marcondes. Assis (SP), Brasil. CEP 19813-550 E-mail: kesleyreticena@hotmail.com
} 


\section{INTRODUÇÃO}

O câncer é uma doença complexa, que demanda cuidados e tratamentos diversos. Durante o tratamento, os pacientes sofrem com possíveis alteraçóes na aparência física, na autoimagem corporal, bem como com eventuais limitaçóes e impedimentos a algumas atividades de rotina, podendo prejudicar sua autoestima e socialização $0^{1-3}$.

Tendo em vista a importância da equipe de enfermagem para pacientes que sofrem com o câncer e a complexidade da atençáo e atuação nessa área, os processos de trabalho desses profissionais devem receber especial atenção ${ }^{2,3}$.

A satisfação na realizaçáo do trabalho qualifica o profissional, levando-o a transmitir confiança ao paciente, prevenir conflitos internos e externos, garantindo melhorias na prestação de serviços e contribuindo para um ambiente agradável. Ademais, proporciona cuidado humanizado e troca de experiências entre profissionais, refletindo no enfrentamento ao tratamento por parte dos pacientes ${ }^{4}$.

Entretanto, profissionais de saúde não estão livres de momentos de estresse, tensão e conflitos entre equipe, familiares e pacientes. Cuidar é algo desgastante, requer mais que conhecimentos e habilidades, exige perseverança e atitudes humanizadas cotidianamente para resgatar as energias positivas e evitar esgotamento profissional ${ }^{5,6}$.

A maioria dos profissionais que auxiliam pacientes com doenças graves, por razôes compreensíveis, mas injustificáveis, evita se envolver, dar atenção às emoçôes dos pacientes, e acaba se distanciando se desvinculando do paciente para prevenir traumas psicológicos que podem ser gerados após a perda ${ }^{5,7}$.

Diante dessa situação, há necessidade de mudanças na prestação de serviços, para que os profissionais de saúde se empenhem em oferecer assistência qualificada, com mais determinação, ética e respeito tanto aos pacientes que lhes são confiados como aos colegas de trabalho, proporcionando, assim, melhor qualidade de vida a ambos ${ }^{2,7}$.

Assim, o objetivo da presente pesquisa é compreender as situaçōes vivenciadas por profissionais de enfermagem que cuidam de pacientes com câncer.

\section{MÉTODO}

Trata-se de pesquisa qualitativa, descritiva e exploratória, que busca compreender o universo de significados, motivos, aspiraçóes, crenças, valores e atitudes, que correspondem ao espaço mais profundo das relaçôes, dos processos e dos fenômenos que não podem ser reduzidos à operacionalização de variáveis ${ }^{8}$.

A pesquisa foi realizada em um hospital do município de Presidente Prudente, SP, Brasil. Foram participantes da pesquisa 12 pessoas do sexo feminino, sendo cinco enfermeiras, cinco técnicas de enfermagem e duas auxiliares de enfermagem.

Para coleta de dados, foi utilizado um roteiro semiestruturado contendo dados de identificação e as seguintes questôes norteadoras: Como é para você trabalhar no cuidado de pacientes que vivenciam o câncer? Que tipo de situaçóes você vivencia durante a prestação de cuidado na unidade oncológica? E como você se sente frente ao cuidado de pessoas com câncer?

A coleta de dados foi realizada por meio de entrevistas aos profissionais de enfermagem em data agendada, conforme seu consentimento e sua disponibilidade, sem prejuízo para a assistência. As entrevistas foram gravadas com auxílio de gravador e transcritas na íntegra por uma das pesquisadoras, sendo que o número de participantes foi definido conforme saturação de dados nas respostas.

Os dados passaram pela análise temática de conteúdo, que ocorreu em três momentos, sendo que, primeiramente, foi feita ordenação dos dados; entáo, foi realizada classificação dos dados com base na estrutura de análise montada por temáticas, separando por categoria ou unidades de sentido todo o material; e terceiro, a análise propriamente dita, consistindo em um processo de codificação, segundo o critério de semelhança temática, marcação das unidades de análise, dividindo em categorias e subcategorias para compreensão e discussão dos dados ${ }^{9}$.

Após análise de dados, emergiram duas categorias "Situações vivenciadas durante a prestação de cuidados na unidade oncológica" e "Sentimentos dos profissionais de enfermagem aos prestarem cuidados ao paciente com câncer".

A presente pesquisa foi aprovada pelo Comitê de Ética e Pesquisa (CEP) da Universidade Paulista (Unip), sob o número de parecer: 1.957.592, e pelo responsável do hospital participante. Antes da realização das entrevistas, foi disponibilizado aos participantes o Termo de Consentimento Livre e Esclarecido (TCLE) em duas vias de igual teor; uma cópia permaneceu com o participante da pesquisa e a outra com o pesquisador. Para manter o anonimato, os sujeitos foram identificados com a letra $\mathrm{E}$, de entrevistados, seguida da numeração de 1 a 12.

\section{RESULTADOS}

Participaram da pesquisa 12 pessoas do sexo feminino, sendo cinco enfermeiras, cinco técnicas de enfermagem e duas auxiliares de enfermagem, na faixa etária de 22 anos a 58 anos. Quanto ao estado civil das entrevistadas, quatro eram solteiras, sete casadas e uma viúva.

As participantes possuíam tempo de experiência em oncologia de dois meses a nove anos e meio. Sete das 
profissionais possuíam especialização em oncologia, enquanto quatro não possuíam, e uma profissional estava cursando.

A seguir, são apresentadas as temáticas que emergiram da análise dos dados.

\section{SITUAÇÕES VIVENCIADAS DURANTE A PRESTAÇÃO DE CUIDADOS NA UNIDADE ONCOLÓGICA}

As 12 pessoas entrevistadas demonstraram sinceridade em suas falas, partilharam experiências vivenciadas durante a prestação de cuidados aos pacientes e relataram situaçóes de dor e alegria.

As situaçóes vivenciadas pelas entrevistadas foram momentos de dores intensas durante o tratamento dos pacientes:

Situação é de muita dor, às vezes, o paciente chega a situaçôes precárias, chegam casos com cuidados paliativos que não dar para fazer nada (E4).

O paciente se queixa de muita dor, às vezes, a gente dá medicaçóes em altas doses para dor e ainda eles queixam de dor, a situaçáo da dor para o paciente em oncologia é um dos suportes que mais tem que intensificar, porque eles sentem bastante dor (E7).

Muitas, muitas situações de dor e sofrimento, é um turbilhão de sentimentos e de situação que a gente passa (E5).

As participantes também relataram dificuldades em acompanhar o processo de morte dos participantes com câncer, especialmente quando esses são jovens, indicando que não estão preparados para enfrentar situaçôes como essas:

O sofrimento é muito grande, quando perdemos um paciente, a gente fica triste (E3).

No começo, foi um pouco meio difícil trabalhar com a morte (E7).

É uma situação dolorosa, você sabe, muitas vezes, que tem paciente, não tem para onde correr, que vai a óbito (E6).

É difícil ver jovens morrendo [...], tentamos mudar o clima de dor e tristeza, mas é muito difícil (E11).

Contudo, algumas entrevistadas relataram que vivenciar o sofrimento diariamente não lhes causa problemas.

Eu não vejo eles como aí coitadinho, tenho dó não, eu vejo como possível cura sempre, nunca, em qualquer estado mesmo, trato da mesma forma, sempre há esperança (E2).

Eu não levo esses sentimentos para casa náo, o que eu posso fazer por eles faço aqui no momento do trabalho (E10).

Eu não vejo problema nenhum não trabalhar aqui (E12).

Procuro náo levar nada do meu trabalho para casa (E8).

Observou-se que a maioria das participantes relatou que os sofrimentos presenciados diariamente não afetam suas vidas pessoais, por possuírem habilidade de distinção e separaçáo entre o pessoal e profissional.

\section{SENTIMENTOS DOS PROFISSIONAIS DE ENFERMAGEM AOS PRESTAREM CUIDADOS AO PACIENTE COM CÂNCER}

Segundo as entrevistadas, ao cuidar dos pacientes com câncer, se sentem realizadas com a profissão, expressaram sentimentos de gratidão e amor pelo trabalho realizado:

Trabalhar aqui é muito positivo, eu não me vejo trabalhar em outro lugar, eu gosto de estar aqui, a gente tem um contato direto com eles (E1).

Eu me identifico com paciente que necessita de um cuidado a mais (E4).

Eu gosto de trabalhar aqui (E12).

Eu amo trabalhar com pacientes oncológicos (E5).

Trabalhar com pacientes oncológicos é a minha vida, é o que eu gosto de fazer, sou muito feliz e realizada (E5).

Algumas participantes também referiram momentos difíceis que já vivenciaram no decorrer dos anos, e dificuldades no início da vida profissional:

No início sim, quando comecei a trabalhar, levava muito para casa, pensando, ficava bem triste (E1).

Não é fácil vivenciar tudo isso, às vezes, acabamos nos abalando com essa situação (E9).

Porém, com as experiências no setor de oncologia, as profissionais aprenderam como proceder, exprimindo o sentimento de segurança em sua atuação:

Hoje, eu lido como pessoa muito melhor, sou muito mais segura se o paciente está sobre cuidados 
paliativos, sente dor, sei até aonde o paciente pode ir com aquela medicação (E7).

É com a vivência mesmo, eu acho que é com a situaçôes que a gente vai vivenciando, você vai conseguindo se encaixar no dia a dia e desempenhar o seu trabalho da melhor forma para eles se sentirem confortáveis e o que a gente preza muito em oncologia é que o paciente se sinta acolhido, se sinta bem (E5).

Mediante as experiências adquiridas durante todo o período de trabalho, evidenciou-se a transformação das profissionais em sua percepçáo de vida e o seu olhar para com os pacientes, buscando sempre aprimorar a assistência prestada. Assim, as participantes relataram segurança na realização dos procedimentos voltados aos pacientes com câncer.

\section{DISCUSSÃO}

Diante da realidade cotidiana dos profissionais perante os pacientes oncológicos, a situação dolorosa é de difícil aceitação, pois envolve sentimentos de empatia e vulnerabilidade, causando momentos de tristeza e decepção por parte dos profissionais ${ }^{10}$.

A literatura demonstra que, para muitos profissionais de saúde, é difícil acompanhar a terminalidade de vida de pacientes, em especial quando o indivíduo é muito jovem e acaba perdendo a vida sem realizar seus objetivos e sonhos, sendo que a morte causa sofrimento e tristeza àqueles que permanecem vivos ${ }^{11,12}$.

Segundo estudos realizados com equipes de enfermagem sobre o enfrentamento da morte, há necessidade de preparação para que os profissionais tenham estrutura psicológica apropriada para enfrentar diversas situaçôes que não tiveram contato durante o período de formação acadêmica, como perda, dor e sofrimento, e para que possam oferecer atendimento humanizado de qualidade ${ }^{10-13}$.

Estudo aponta que, ao vivenciar diariamente sofrimento e declínio dos pacientes, os trabalhadores experienciam sobrecarga emocional, dificultando a divisão entre profissional e pessoal, ocasionando sofrimentos a todos envolvidos no processo. Contudo, conforme as experiências adquiridas, os profissionais se dão conta de que a morte é um processo natural e fisiológico do ser humano ${ }^{14}$.

Conforme observado nas falas, as profissionais de enfermagem no setor de oncologia expressaram gratidão e satisfação no trabalho realizado. Esses dados corroboram os resultados de pesquisas que apontam que, mesmo em momentos de dificuldades, profissionais de enfermagem do setor de oncologia sentem grande satisfaçáo por realizar seu trabalho com amor, podendo ajudar aqueles que se encontram em situação difícil, mantendo vínculo com os pacientes e familiares, vivenciando suas dores e os fortalecendo com motivaçóes positivas, ofertando um cuidado digno e adequado para a qualidade de $\mathrm{vida}^{12}$.

Estudos demonstram que profissionais de oncologia vivem satisfeitos e gostam do trabalho prestado aos pacientes oncológicos e que é possível realizar cuidado exigente, obtendo satisfação à medida que se ama o que faz, se dedicando sem reserva às necessidades do outro. Quando se doa carinho, o clima se transforma, o relacionamento humano e o amor renascem por dentro, por meio de sentimentos de gratidão e esperança ${ }^{15}$.

Outros estudos mostram que profissionais de enfermagem enfrentam dificuldades que lhes proporcionam insatisfação, causando estresse e outros tipos de doenças ${ }^{4}$ Cuidar é algo cansativo, podendo sobrecarregar emocionalmente o profissional e, caso este não possua estratégias de enfrentamento, ocorre o adoecimento $^{5,7}$.

Nem todos possuem capacidades de trabalhar no setor de oncologia. Estudos relatam que é preciso, além da capacitação, que esses profissionais convivam com os pacientes, nas fases da doença, de modo, natural e humano, expressando sentimentos, dando apoio nos momentos de fragilidade, sendo suporte, e sendo uma pessoa em quem o doente possa confiar ${ }^{12}$.

Para promover o alívio da dor, é necessário, em primeiro lugar, a açáo medicamentosa e, posteriormente, a alegria e atenção; ou seja, ofertando uma assistência articulada, buscando atingir o objetivo principal, fazendo com que os pacientes amenizem as suas dores. Nesse sentido, os profissionais transmitem e executam seu trabalho com segurança e determinação, visualizando efeitos esperados do cuidado ofertado ao paciente, atentando sempre para situaçóes que possam ocasionar aumento de dores, como desamparo, medo, assistência náo humanizada e desconforto no leito ${ }^{16,17}$

Profissionais de saúde vivem lutos cotidianos sem direito de expressão de suas dores, emoções e sentimentos, tendo que buscar estratégias de enfrentamento. Portanto, a formação e a aceitação de cada profissional podem levar ao aumento de adoecimento e a casos de depressão, em razão de sentimento de impotência, frustração e revolta. Isso vem contribuindo para aumento da síndrome de Burnout, exaustão emocional, despersonalizaçâo e diminuição da realização pessoal entre profissionais ${ }^{2,7}$.

Mesmo em meio aos desafios do cuidado, é possível ser satisfeito com o trabalho realizado, obter inspiração positivas, aprender a cultivar os valores da vida, amar e sorrir, transmitindo sempre esperança para aqueles que necessitam. Os trabalhos realizados em equipe com trocas de experiência são um dos motivos que podem elevar 
o grau de satisfação do profissional e contribuir para melhorias na prestação de cuidados aos pacientes ${ }^{4}$.

A realização da presente pesquisa possibilitou a compreensão das situações vivenciadas pela equipe de enfermagem no setor de oncologia, contudo, tem-se a limitaçáo de que a pesquisa realizada envolveu somente a equipe de enfermagem. Sugere-se a realizaçáo de novas pesquisas abrangendo toda a equipe multidisciplinar, podendo, assim, obter conhecimento maior sobre as diversas situaçóes vivenciadas e o modo de enfrentamento presentes na realizaçáo do serviço.

\section{CONCLUSÃO}

Os resultados alcançados permitiram compreender as situaçóes vivenciadas por profissionais de enfermagem que cuidam de pacientes com câncer, as quais são compostas de dificuldades e satisfaçáo. Essa compreensão é de extrema importância para a enfermagem no campo da oncologia, na medida em que desnuda situaçóes vivenciadas no cotidiano da prática assistencial dos profissionais que atuam na especialidade.

As situaçóes vivenciadas pelas entrevistadas foram momentos de dores intensas durante o tratamento dos pacientes, e dificuldade em acompanhar o processo de morte. Contudo, as participantes também demonstraram sentimentos de satisfação e gratidão com o trabalho realizado. Considerando-se que a forma como os profissionais elaboram e ressignificam esses sentidos e sentimentos pode influenciar diretamente na qualidade da assistência, é relevante compreender o seu preparo técnico e emocional.

Os resultados desta pesquisa indicam que as profissionais estấo preparadas profissional e emocionalmente para o atendimento a esse grupo de paciente, pois, em sua maioria, têm experiência e possuem especialização em oncologia. Apesar disso, é necessário que haja formação permanente e continuada, tendo em vista que sempre há inovações na ciência buscando melhoria na assistência.

\section{CONTRIBUIÇÕES}

Géssica Fernanda Rodrigues Monção e Estela Maris Ribeiro Cardoso foram responsáveis pela concepção da pesquisa, trabalho de campo e redaçáo do artigo. Kesley de Oliveira Reticena, Maria Fernanda Pereira Gomes e Lislaine Aparecida Fracolli orientaram a pesquisa e fizeram a revisão do manuscrito. Todas as autoras aprovaram versão final do artigo.

\section{DECLARAÇÃO DE CONFLITO DE INTERESSES}

Nada a declarar.

\section{FONTES DE FINANCIAMENTO}

Não há.

\section{REFERÊNCIAS}

1. Instituto Nacional de Câncer José Alencar Gomes da Silva. Tratamento do câncer [Internet]. Rio de Janeiro: INCA; 2017. [acesso 2018 Mar 23]. Disponível em: http://www2.inca.gov.br/wps/wcm/connect/ tiposdecancer/site/home/infantil.

2. Silva MM, Santanda NGM, Santos MC, et al. Cuidados paliativos na assistência de alta complexidade em oncologia: percepção de enfermeiros. Esc Anna Nery. 2015;19(3):460-466. doi: http://dx.doi. org/10.5935/1414-8145.20150061.

3. Souza GRM, Cazola LHO, Oliveira SMVL. Atuação dos enfermeiros da estratégia saúde da família na atenção oncológica. Esc. Anna Nery. 2017;21(4):e20160380. doi: http://dx.doi.org/10.1590/2177-9465-ean-2016-0380.

4. Bordignon M, Monteiro MI, Mai S, et al. Satisfação e insatisfação no trabalho de profissionais de enfermagem da oncologia do Brasil e Portugal. Texto Contexto Enferm. 2015;24(4):925-933. doi: http://dx.doi. org/10.1590/0104-0707201500004650014.

5. Corrêa RZA, Souza MS, Baptista MN. Vulnerabilidade ao estresse no trabalho e qualidade de vida de enfermeiros. Psicol Argum. 2013;31(75):599-606. doi: http://dx.doi. org/10.7213/psicol.argum.31.075.DS02.

6. Longuiniere ACF, Yarid SD, Silva ECS. Influência da religiosidade/espiritualidade do profissional de saúde no cuidado ao paciente crítico. Rev Cuid. 2018;9(1):19611972. doi: https://doi.org/10.15649/cuidarte.v9i1.413.

7. Coblinski DR, Wisniewski D, Hey A. Síndrome de burnout em profissionais da equipe de enfermagem. Rev Uninga [Internet]. 2018 [citado 2018 Mar 16];45(1):2733. Disponível em: http://revista.uninga.br/index.php/ uninga/article/view/1236.

8. Minayo MCS. (organizadora). Pesquisa social: teoria, método e criatividade. 30. ed. Petrópolis: Vozes; 2011.

9. Campos CJG. Método de análise de conteúdo: ferramenta para a análise de dados qualitativos no campo da saúde. Rev Bras Enferm. 2004;57(5):611-614. doi: http:// dx.doi.org/10.1590/S0034-71672004000500019.

10. Marchi JA, Paula, CC, Girardon-Perlini, NMO, et al. Significado de ser-cuidador de familiar com câncer e dependente: contribuiçóes para a paliação. Texto Contexto Enferm. 2016;25(1):e0760014. doi: http:// dx.doi.org/10.1590/0104-07072016007600014.

11. Bastos RA, Lamb FA, Quintana AM, et al. Vivências dos enfermeiros frente ao processo de morrer: uma metassíntese qualitativa. Revista Portuguesa de Enfermagem de Saúde Mental. 2017 Jun;(17):58-64. doi: http://dx.doi.org/10.19131/rpesm.0184. 
12. Andrade FLM, Silva MES, Brito DTF, et al. Dor oncológica: manejo clínico realizado por enfermeiros. Revista de Iniciação Cientifica da Universidade Vale do Rio Verde. 2018;8(1):3-16.

13. Ayala ALM, Felício ACR, Pachão J. Sofrimento dos profissionais que atuam no setor de oncologia em um hospital público de Joinville, SC. Rev Aten Saúde. 2017;15(51):106-117. doi: http://dx.doi.org/10.13037/ ras.vol15n51.4376.

14. Lima ABS, Oliveira LP, Sá KVCS, et al. Sentimentos e percepçóes da enfermagem frente ao processo de morte e morrer: revisão integrativa. Rev Pesq Saúde. 2016;17(2):116-121.

15. Trindade LL, Bordignon M, Ferraz L. Satisfação no trabalho em oncologia: uma revisão integrativa da literatura. J Nurs Health. 2014;4(2):177-85. doi: http:// dx.doi.org/10.15210/jonah.v4i2.3868.

16. Alves VS, Santos TS, Trezza MCSF, Santos RM, Monteiro FS. Conhecimento de profissionais da enfermagem sobre fatores que agravam e aliviam a dor oncológica. Rev Bras Cancerol. 2011;57(2):199-206.

17. Xavier SS, Anjos KF, Sampaio KCP, et al. Cuidado humanizado do enfermeiro ao paciente oncológico fora de possibilidade de cura. Rev. Saúde.Com. 2017;13(4):10441054. doi: http://dx.doi.org/10.22481/rsc.v13i04.519. 